\title{
Analysis on the Countermeasures of Building the Talent Team of the Elderly Care Service Industry in Sichuan Province
}

\author{
Li Bing ${ }^{1,}$, Tian Yü ${ }^{2, b}$ \\ ${ }^{1}$ School of Public Management, University of Electronic Science and Technology, Chengdu, \\ Sichuan, China \\ ${ }^{2}$ School of Public Management, University of Electronic Science and Technology, Chengdu, \\ Sichuan, China \\ a1065810507@qq.com, b451328932@qq.com
}

\begin{abstract}
Keywords: Sichuan province; elderly service industry; talent team construction; countermeasure analysis
\end{abstract}

\begin{abstract}
Sichuan Province, as the second largest province in China in terms of aging population, urgently needs to accelerate the development of the elderly care service industry. The elderly population in Sichuan Province has a strong demand for care for the aged in terms of life care, rehabilitation care and spiritual comfort. Therefore, the cultivation of socialized, professional, and vocational nursing care service talents is the key factor to ensure that the elderly receive diversified quality care services. Starting with the actual background of the construction of the talent service team for the aged in Sichuan Province, this paper analyzes the current situation and the experience at home and abroad, to find out the current problems and causes of the construction of the talents service system in Sichuan's aged care industry, and proposes suggestions and countermeasures for the development talents for the elderly service industry in Sichuan Province.
\end{abstract}

\section{Introduction}

The 19th National Party Congress writes the following into report: "proactively responding to the aging of the population, building a policy system and social environment for nursing the elderly, filial piety, and respecting the elderly, advancing the combination of medical care and health care, and accelerating the development of old-age businesses and industries". Old age businesses and industries have become the focus of concern at the national level. By the end of 2016, the population of Sichuan Province aged 60 years or over reached 17.39 million, accounting for $21.1 \%$ of the permanent population, which was 4.4 percentage points higher than the national total. Among them, the population aged 65 and over reached 11.38 million. According to statistics published by different areas in China, the proportion of elderly aged over 65 in Sichuan Province ranks first in the country. With the advent of an ageing population,the shortage of talent is one of the major bottlenecks restricting the development of China's elderly service industry. The elderly population in Sichuan Province needs a variety of care services, and the demand for health care and daily care is more rigid. The construction of the talent team for the aged care industry in Sichuan is in urgent need of training multi-dimensional and planned teams of aged care and management personnel with appropriate age gradients, reasonable scale ratios, and requirements in line with professional competence, psychological quality, moral cultivation, and cultural level.

\section{Problems and Causes of the Talent Team Construction in the Elderly Care Services in Sichuan Province}

\subsection{Talent management is under management of multiple government departments, and the allocation of various resources is limited}

The Working Committee office on the Aging is the deputy director of the Ministry of Civil Affairs. However, elderly care services are now not just the responsibility of the civil affairs and medical 
committees in the traditional sense, but also related to culture, education and so on. The current Working Committee office on the Aging can play a supervisory role in a simple job or project. However, there are indeed serious difficulties in systematically issuing, supervising and evaluating the industry, especially the deployment of personnel resources and financial resources.

\subsection{There is a difference between the training of elderly care personnel and the actual needs of the elderly}

First, the training level is low. At present, most elderly care workers in Sichuan Province graduate from junior high school. The education level is limited, and there is lack professional knowledge and skills. Among them, only rarely hold qualification certificates. The employees are mostly rural migrant workers and urban unemployed workers. The overall age is too old.

Second, there is still a certain gap between the knowledge structure and professional skills of the elderly care providers and the needs of industry enterprises. The existing old-age caregivers mainly provide the elderly with basic daily care, and it is difficult to provide the elderly with the diversified, humanized and personalized services they need such as psychological care and spiritual comfort.

Third, the form of training professionals is single, focusing on theory and practice. At present, the teaching staffs training professional personnel serving Sichuan's elderly care service are a few, and practice teaching in the professional personnel training system accounts for a relatively low proportion. At the same time, there are few traineeships and internships provided by the institutions, and the length of the internships is short and the number of applications is small.

\subsection{The design of the service guarantee mechanism makes it difficult to attract and retain talents in the elderly care service industry}

First, the elderly care service features low wages, labor intensity, and less benefits. Besides, in traditional concepts, there are certain derogatory views on nursing care workers and "servers", and the unsatisfied status of social status increases the mobility of nursing care workers.

Second, due to the lack of the mechanism and policy system on matched professional qualification certification, job title assessment, and salary adjustment, the development environment of the industry is unstable, and the supply of professionals is imbalanced and broken [1].

Practitioners generally have low satisfaction with their work. For example, a sample survey conducted by the Beijing Institute of Social Management on provinces and cities found that nearly $30 \%$ of people are not satisfied with their income [2].

\subsection{Related laws and regulations are not perfect and there is lack of effective market supervision}

The elderly care service industry has not established a strict entry and exit mechanism and lacks sound industry management laws and regulation. The quality of elderly care service personnel is uneven, and there is widespread distrust of people for the employees. Insults and violent attacks on the elderly occurs commonly. Under such environment, service receivers and their children are alert to the practitioners, which affects the development of the elderly care service industry in Sichuan.

\section{Analysis of the development path of the talent team in the elderly care service industry in Sichuan province}

\subsection{Upgrading aging offices' ability to integrate resources to coordinate management of various issues}

It is suggested that the leaders of the municipal party committee should be set up to take charge of the leadership, allocate various departments and promote the concept and tendency of serving the elderly from all walks of life. Although some of our organizational reforms are top-down, if taking a close look at the achievements we have made in the past four decades of reform and opening up, many major reforms have created a mechanism from the bottom up to force the smooth implementation of the work. 


\subsection{Focusing on improving professional quality and professional skills, nurturing comprehensive elderly caregivers}

In order to cultivate comprehensive old-age service providers, first, it should strengthen the innovation and integration of services for elder service education, school-enterprise matchmaking, and internationalization of education, and it requires professional colleges, trade associations, and vocational training institutions in the elderly care industry to have deep cooperation.Second, by taking into account that mostly diseases for the elderly are chronic diseases, Sichuan Province should also make rational use of the province's rich resources of traditional Chinese medicine and give full play to the advantages of Chinese medicine in the use for the elderly to maintain physical and mental health, prevent diseases and treat disease.

\subsection{To build a service-graded model for the training of the aged according to the needs of the elderly}

According to the difference in the needs of the elderly, Japan divides the service personnel for the aged into welfare and social welfare staff. The welfare services are divided into three grades, and different grades provide different contents and levels [3]. Sichuan's provision system for elderly care services should refer to this concept and categorize and train specialized talents according to the demand-oriented classification.

First, the elderly care service skills training institutions should adopt short-term training methods to improve the skills of existing industry personnel. Training practitioners should provide daily care for the elderly and master the emergency treatment of common situations of the elderly.

Second, the institutions of higher vocational education should aim at cultivating nurses for the elderly, highlight the cultivation of practical skills for students, and train applied and skilled nursing care personnel.

Third, colleges and universities and research institutes should strengthen the building of nursing care professional services, with the goal of training research-oriented, innovative, and educational nursing care professionals. At the same time, scientific research in this field should be carried out to strengthen the construction of teacher training, curriculum construction and teaching materials for the nursing care professional, and to improve the industry's specialization and knowledge level and improve the image of the industry [4].

\subsection{Innovate talent system and promote the development of talents in the service industry}

First, the government can allow and encourage elderly care service agencies to properly set up higherlevel professional skills and work-related skills positions to open career development channels for employees to gain job promotion opportunities.

Second, the government must especially support and give corresponding preferential policies for talents of both medical care and health care. Specifically, it includes investment in graduates and talents; job subsidies, job titles, and special awards for those who have long-term adherence to services and special contributions.

\subsection{Enhance the Standardized Management of Talents Work According to Law}

First, to strengthen the relevant legislation of the elderly care service industry, standardize the management of employees in the industry, and ensure the legal system, standardization, and orderly development of the building of a talent service team for the aged.

Second, to develop a unified industry standard which ensures the standardization of elderly care services. Establish a strict access mechanism, formulate a comprehensive supervision system, improve industry entry barriers, and conduct strict qualification audits.

Third, the practitioners will be regularly audited, and the service providers will be included in the main body of performance evaluation to evaluate the service quality of practitioners. 


\subsection{Relying on major projects to speed up the incubation and growth of nursing professionals}

First, to build a number of education and teaching bases for provincial and municipal elderly care workers and managers. Actively develop and rely on qualified universities, research institutes, training organizations, and elderly service organizations to carry out training.

Second, to develop the job rotation projects of the medical staff of public hospitals. Based on the current situation in the difficulties in recruitment for private operated elderly care service institutions, it is hoped that the medical staff of public hospitals can take a temporary post in private organizations and nursing institutions as a long-term mechanism.

\section{References}

[1] Deng Dasong, Li Yujiao. The combination of medical care and health care: Institutional rationality, supply and demand dilemma, and model innovation [J]. Journal of Xinjiang Normal University (Philosophy and Social Science Edition). 2018, 39(1): 107-114.

[2] Zhen Bingliang, Liu Jianhua. Study on the Construction of Talent Service and Talent Team in China, Chinese Civil Policy. 2014 (07): 29-30.

[3] Cui Wei, Zhou Yue. The abroad experience of how to train professional pension service talent[N]. China Social News, 2013-12-02(04).

[4] Wang Sha, He Guoping, Yao Juqin, Luo Yan. Personnel training in health service industry and pension service industry $[\mathrm{J}]$. Talent cultivation in health service industry and pension service industry, 2016: 1767-1769. 